\title{
On the Maximum Harary Spectral Radius of Graphs with Given Matching Number
}

\author{
Xin Luo, Wenshui Lin*, Zhixi Wu, Linshan Huang and Qi'an Chen \\ School of Information Science and Engineering, Xiamen University, Xiamen, P.R. China \\ ${ }^{*}$ Corresponding author
}

\begin{abstract}
The Harary spectral radius was proved to be able to produce fair QSPR models for the boiling points, molar heat capacities, vaporization enthalpies, refractive indices and densities for $\mathrm{C}_{6}-\mathrm{C}_{10}$ alkanes. Hence this structure-descriptor has recently attracted attention of chemists and mathematicians. [Ars Combin. 131 (2017) 23] and [Appl. Math. Comput. 266 (2015) 937] considered the maximum Harary spectral radius of graphs with given matching number. However, the results are wrong or incomplete. This paper gives an almost complete solution to the problem.
\end{abstract}

Keywords-Harary matrix; spectral radius; matching number; extremal graph

\section{INTRODUCTION}

We consider non-trivial connected simple graphs only. Such a graph will be denoted by $G=(V, E)$, where $V=\left\{v_{1}, v_{2}, \cdots, v_{n}\right\}$ and $E=E(G)$ are the vertex set and edge set of $G$, respectively. $\bar{G}$ will denote the complement of $G$. Let $G_{1} \cup G_{2}$ be the vertex-disjoint union of the graphs $G_{1}$ and $G_{2}$, and $G_{1} \vee G_{2}$ the graph obtained from $G_{1} \cup G_{2}$ by joining each vertex of $G_{1}$ to each vertex of $G_{2}$.

A matching of $G$ is a set of pairwise non-adjacent edges. A maximum matching is one which contains as many edges as possible. The cardinality of a maximum matching of $G$ is called the matching number of $G$

The Harary matrix $R D(G)$ of $G$, which is also known as the reciprocal distance matrix [1], is an $n \times n$ matrix whose $(i, j)$-entry $R D_{i j}$ is equal to $1 / d_{i j}$ if $i \neq j$ and 0 otherwise, where $d_{i j}$ is the distance between $v_{i}$ and $v_{j}$ in $G$. Since $R D(G)$ is non-negative and symmetric, its eigenvalues are all real. The spectral radius (the largest eigenvalue) of $R D(G)$, denoted by $\rho(G)$, is called the Harary spectral radius of $G$. The Harary index of $G$ is defined as $\sum_{i<j} R D_{i j}$, and the Harary energy of $G$ is the sum of the absolute values of all eigenvalues of $R D(G)$.

Ivanciuc et al. [2] showed that, the Harary spectral radius is able to produce fair quantitative structure - property relationships (QSPR) models for the boiling points, molar heat capacities, vaporization enthalpies, refractive indices and densities for $\mathrm{C}_{6}-\mathrm{C}_{10}$ alkanes. Hence this structure-descriptor has recently attracted attention of chemists and mathematicians. The lower and upper bounds of the Harary spectral radius of the Harary matrix, and the Nordhaus-Gaddum-type results for it were obtained in [3, 4]. Cui and Liu [5] proposed some more results about the eigenvalues of Harary matrices; also, they got some bounds of the Harary energy. Some lower and upper bounds for the Harary energy of connected $(n, m)$-graphs were obtained in [6]. One can also refer to the book [7] for some properties and applications of Harary index and its variants.

Zhu et al. [8] considered the maximum Harary spectral radius of graphs with given matching number $p$, and claimed that, the extremal graph is $G=K_{1} \vee\left(K_{2 p-1} \cup \overline{K_{n-2 p}}\right)$ if $p<\lfloor n / 2\rfloor$, where $K_{a}$ denotes the complete graph of order $a$. However, the result is wrong. Independently, Huang et al. [9] considered the same problem, and proved the following result.

Theorem 1.1 [9]. Let $G$ be a graph on $n$ vertices with matching number $p$ which has the maximum Harary spectral radius. Then

(1) If $1 \leq p \leq\lfloor n / 3\rfloor$, then $G=K_{p} \vee \overline{K_{n-p}}$;

(2) If $\lfloor n / 3\rfloor<p<\lfloor n / 2\rfloor$, then $G=K_{1} \vee\left(K_{2 p-1} \cup \overline{K_{n-2 p}}\right)$ or $G=K_{p} \vee \overline{K_{n-p}}$;

(3) If $p=\lfloor n / 2\rfloor$, then $G=K_{n}$.

Hence, for the case $\lfloor n / 3\rfloor<p<\lfloor n / 2\rfloor$ the extremal graph has not been characterized completely. In the rest of this paper, we let $G_{1}=K_{1} \vee\left(K_{2 p-1} \cup \overline{K_{n-2 p}}\right)$ and $G_{2}=K_{p} \vee \overline{K_{n-p}}$. For completeness, one needs to know when $\rho\left(G_{1}\right)>\rho\left(G_{2}\right)$ holds. In Section II, we will prove that, $\rho\left(G_{1}\right)>\rho\left(G_{2}\right)$ if $p>p_{0}(n)=\left(24 n+9-\sqrt{48 n^{2}-8 n-7}\right) / 44$.

\section{MAIN RESULTS}

Since $R D(G)$ is real, non-negative, and irreducible, from the Perron-Frobenius theorem, $\rho(G)$ is positive, simple, and there is a unique positive unit eigenvector $X$ corresponding to $\rho(G)$, which is called the Perron vector of $R D(G)$. That is, 
$\rho(G)=\max _{\|Y\|=1} Y^{\prime} R D(G) Y=X^{\prime} R D(G) X$. Moreover, we have $\rho(G) X=R D(G) X$.

Let $\rho_{1}=\rho\left(G_{1}\right)$, and $\rho_{2}=\rho\left(G_{2}\right)$. Let $J_{k \times l}$ be the $k \times l$ matrix whose entries are all equal to 1 , and $I_{k}$ the $k \times k$ unit matrix. It is easily seen that,

$$
R D\left(G_{1}\right)=\left(\begin{array}{ccc}
(J-I)_{(2 p-1) \times(2 p-1)} & J_{(2 p-1) \times 1} & \frac{1}{2} J_{(2 p-1) \times(n-2 p)} \\
J_{1 \times(2 p-1)} & 0_{1 \times 1} & J_{1 \times(n-2 p)} \\
\frac{1}{2} J_{(n-2 p) \times(2 p-1)} & J_{(n-2 p) \times 1} & \frac{1}{2}(J-I)_{(n-2 p) \times(n-2 p)}
\end{array}\right),
$$

and

$$
R D\left(G_{2}\right)=\left(\begin{array}{cc}
(J-I)_{p \times p} & J_{p \times(n-p)} \\
J_{(n-p) \times p} & \frac{1}{2}(J-I)_{(n-p) \times(n-p)}
\end{array}\right) .
$$

Let

$$
A=\left(\begin{array}{cc}
(J-I)_{2 p \times 2 p} & \frac{1}{2} J_{2 p \times(n-2 p)} \\
\frac{1}{2} J_{(n-2 p) \times 2 p} & \frac{1}{2}(J-I)_{(n-2 p) \times(n-2 p)}
\end{array}\right),
$$

and $\rho_{3}=\rho(A)$. Since

$$
B=R D\left(G_{1}\right)-A=\left(\begin{array}{ccc}
0_{(2 p-1) \times(2 p-1)} & 0_{(2 p-1) \times 1} & 0_{(2 p-1) \times(n-2 p)} \\
0_{1 \times(2 p-1)} & 0_{1 \times 1} & \frac{1}{2} J_{1 \times(n-2 p)} \\
0_{(n-2 p) \times(2 p-1)} & \frac{1}{2} J_{(n-2 p) \times 1} & 0_{(n-2 p) \times(n-2 p)}
\end{array}\right)
$$

is non-negative, $\rho_{1}>\rho_{3}$ holds immediately from the PerronFrobenius theorem.

To compare $\rho_{3}$ and $\rho_{2}$, we will use a novel technique that involves comparing the largest roots of two certain polynomials. Let $f(x)$ be a polynomial, $\operatorname{deg}(f)$ and $\lambda_{1}(f)$ will denote the degree and the largest root of $f(x)$, respectively.

Lemma 2.1. Let $f(x)$ and $g(x)$ be two monic polynomials with real roots, such that $\operatorname{deg}(f) \geq \operatorname{deg}(g)$ and $f(x)=q(x) g(x)+a x+b$, where $a \neq 0, b$ are two real numbers, $q(x)$ is a monic polynomial with $\lambda_{1}(q)<\lambda_{1}(g)$, and $f(x), g(x)$ increase monotonically in $[-b / a,+\infty)$. Then we have

(1) If $a>0$, then $\lambda_{1}(f) \geq \lambda_{1}(g)$ iff $g(-b / a) \geq 0$, with the equality iff $g(-b / a)=0$.

(2) If $a<0$, then $\lambda_{1}(f) \geq \lambda_{1}(g)$ iff $g(-b / a) \leq 0$, with the equality iff $g(-b / a)=0$.
Proof. (1) If $g(-b / a) \geq 0$, then $g(x)>0$ for $x>-b / a$, since $g(x)$ increases monotonically in $[-b / a,+\infty)$. Hence $\lambda_{1}(g) \leq-b / a$, and $f\left(\lambda_{1}(g)\right)=a \lambda_{1}(g)+b \leq 0$, which implies $\lambda_{1}(f) \geq \lambda_{1}(g)$, with the equality iff $f(-b / a)=0$.

Conversely, if $g(-b / a)<0$, then $\lambda_{1}(g)>-b / a$. Since $\lambda_{1}(q)<\lambda_{1}(g), q(x)>0$ for $x \geq \lambda_{1}(g)$. Hence we have $f(x)=q(x) g(x)+a x+b>0$ for all $x \geq \lambda_{1}(g)$, which implies $\lambda_{1}(f)<\lambda_{1}(g)$.

(2) Analogously to Case (1).

Lemma 2.2. $\rho_{3} \geq \rho_{2}$ iff $p \geq p_{0}(n)$, with the equality iff $p_{0}(n)$ is an integer and $p=p_{0}(n)$.

Proof. Let $X=\left(x_{1}^{2 p}, x_{2}^{n-2 p}\right)^{\prime}$ be the Perron vectors of $A$, where $z^{k}$ denotes $k$ consecutive $z$ 's. From $\rho_{3} X=A X$ we have

$$
\begin{aligned}
& \left\{\begin{array}{l}
\rho_{3} x_{1}=(2 p-1) x_{1}+\frac{1}{2}(n-2 p) x_{2} \\
\rho_{3} x_{2}=p x_{1}+\frac{1}{2}(n-2 p-1) x_{2}
\end{array}\right. \\
& \Rightarrow\left\{\begin{array}{l}
2\left(\rho_{3}-2 p+1\right) x_{1}=(n-2 p) x_{2} \\
\left(2 \rho_{3}-n+2 p+1\right) x_{2}=2 p x_{1}
\end{array}\right. \\
& \Rightarrow \frac{2 x_{1}}{x_{2}}=\frac{n-2 p}{\rho_{3}-(2 p-1)}=\frac{2 \rho_{3}-(n-2 p-1)}{p}
\end{aligned}
$$

$$
\Rightarrow 2 \rho_{3}^{2}-(n+2 p-3) \rho_{3}+\left(p n-2 p^{2}-n+1\right)=0 \text {. }
$$

Since $A$ is nonnegative and irreducible, $\rho_{3}$ is bounded by the minimum and maximum row sums of $A$. Noting $p \leq n / 2$, we immediately have $\rho_{3} \geq(n-1) / 2>(n+2 p-3) / 4$. Hence $\rho_{3}$ is the larger (real) root of the quadratic function $f(x)=x^{2}-(n+2 p-3) x / 2+\left(p n-2 p^{2}-n+1\right) / 2$.

Similarly, $\rho_{2}$ is the larger root of the quadratic function $g(x)=x^{2}-(n+p-3) x / 2+\left(p^{2}-p n-n+1\right) / 2$.

Let $x_{0}=2 n-3 p \quad$ and $r(x)=p\left(x_{0}-x\right) / 2$. Then $f(x)=g(x)+r(x)$. Since $p<n / 2$, it is easily confirmed that, $x_{0}>(n+2 p-3) / 4>(n+p-3) / 4$. Hence $f(x)$ and $g(x)$ increase monotonically in $\left[x_{0},+\infty\right)$. From Lemma 2.1 (2) we have $\rho_{3} \geq \rho_{2}$ iff $g\left(x_{0}\right) \leq 0$, with the equality iff $g\left(x_{0}\right)=0$. By elementary computations we have

$$
g\left(x_{0}\right) \leq 0 \Leftrightarrow x_{0}^{2}-(n+p-3) x_{0} / 2+\left(p^{2}-p n-n+1\right) / 2 \leq 0
$$




$$
\begin{aligned}
& \Leftrightarrow 22 p^{2}-(24 n+9) p+6 n^{2}+5 n+1 \leq 0 \\
& \Leftrightarrow p \geq p_{0}(n)=\left(24 n+9-\sqrt{48 n^{2}-8 n-7}\right) / 44 .
\end{aligned}
$$

The proof is thus completed.

Theorem 2.3. If $p>p_{0}(n)$, then $\rho_{1}>\rho_{2}$.

Proof. Immediate from $\rho_{1}>\rho_{3}$ and Lemma 2.2.

\section{FURTHER DISCUSSIONS}

Theorem 2.3 partially solves the comparison between $\rho_{1}=\rho\left(K_{1} \vee\left(K_{2 p-1} \cup \overline{K_{n-2 p}}\right)\right) \quad$ and $\quad \rho_{2}=\rho\left(K_{p} \vee \overline{K_{n-p}}\right)$. However, the case $\lfloor n / 3\rfloor<p<p_{0}(n)$ remains unknown. Naturally, we propose the following conjecture.

Conjecture 3.1. There exists a positive integer $p_{1}(n)$ with $\left\lceil p_{0}(n)\right\rceil-p_{1}(n) \leq 1$ such that, $\rho_{1} \geq \rho_{2}$ iff $p \geq p_{1}(n)$, with the equality iff $p=p_{1}(n)$.

To support the above conjecture, we conducted a numerical experiment, which confirms the conjecture for $n \leq 1500$.

To prove or disprove Conjecture 3.1 , one may need to estimate the gap $\rho_{1}-\rho_{3}$ precisely. However, at the present we only have the following result, which is quite trivial.

Lemma 3.2. $\rho_{1}-\rho_{3} \leq(n-2 p) / 2$, and $\rho_{1}-\rho_{3}<n / 6$ if $p>n / 3$.

\section{Proof. Since}

$$
B=R D\left(G_{1}\right)-A=\left(\begin{array}{ccc}
0_{(2 p-1) \times(2 p-1)} & 0_{(2 p-1) \times 1} & 0_{(2 p-1) \times(n-2 p)} \\
0_{1 \times(2 p-1)} & 0_{1 \times 1} & \frac{1}{2} J_{1 \times(n-2 p)} \\
0_{(n-2 p) \times(2 p-1)} & \frac{1}{2} J_{(n-2 p) \times 1} & 0_{(n-2 p) \times(n-2 p)}
\end{array}\right)
$$

is nonnegative and irreducible, $\rho(B)$ is bounded by the minimum and maximum row sums of $B$, hence $\rho(B) \leq(n-2 p) / 2$. From the Courant-Weyl inequality [10] we have $\rho_{1}-\rho_{3} \leq \rho(B) \leq(n-2 p) / 2$, and $\rho_{1}-\rho_{3}<n / 6$ if $p>n / 3$.

\section{ACKNOWLEDGMENT}

This project is supported by the National Natural Science Foundation of China (No. 11771362).

\section{REFERENCES}

[1] O. Ivanciuc, T. S. Balaban, and A. T. Balaban, "Design of topological indices. Part 4. reciprocal distance matrix, related local vertex in variants and topological indices," J. Math. Chem., vol. 12, pp. 309-318, 1993.

[2] O. Ivanciuc, T. Ivanciuc, and A. T. Balaban, "Quantitative structureproperty relationship evaluation of structural descriptors derived from the distance and reverse Wiener matrices," Internet Eletron. J. Mol. Des., vol. 1, pp. 467-487, 2002.
[3] K. C. Das, "Maximum eigenvalues of the reciprocal distance matrix," J. Math. Chem., vol. 47, pp. 21-28, 2010.

[4] B. Zhou, and N. Trinajstć, "Maximum eigenvalues of the reciprocal distance matrix and the reverse Wiener matrix", Int. J. Quantum Chem., vol. 108, pp. 858-864, 2008.

[5] Z. Cui, and B. Liu, "On Harary matrix, Harary index and Harary energy," MATCH Commun. Math. Comput. Chem., vol. 68, pp. 815$823,2012$.

[6] A. D. Gungor, and A. S. Çevik, "On the Harary energy and Harary Estrada index of a graph," MATCH Commun. Math. Comput. Chem., vol. 64, pp. 280-296, 2010.

[7] K. Xu, K. C. Das, and N. Trinajstić, The Harary Index of a Graph, Springer, 2015.

[8] Z. Zhu, H. Wei, X. Ma, T. Wang, and W. Zhu, "On Harary spectral radius with given parameters," Ars Combin., vol. 131, pp. 23-30, 2017.

[9] F. Huang, X. Li, and S. Wang, "On graphs with maximum Harary spectral radius,” Appl. Math. Comput., vol. 266, pp. 937-945, 2015.

[10] D. Cvetković, M. Doob, and H. Sachs, Spectra of Graphs-Theory and Applications, 3rd ed., Johann Ambrosius Barth Verlag, 1995. 